\title{
基于 BIM 技术的绿色建筑设计探析
}

\section{Analysis of Green Building Design Based on BIM Technology \\ 魏明耀}

Mingyao Wei

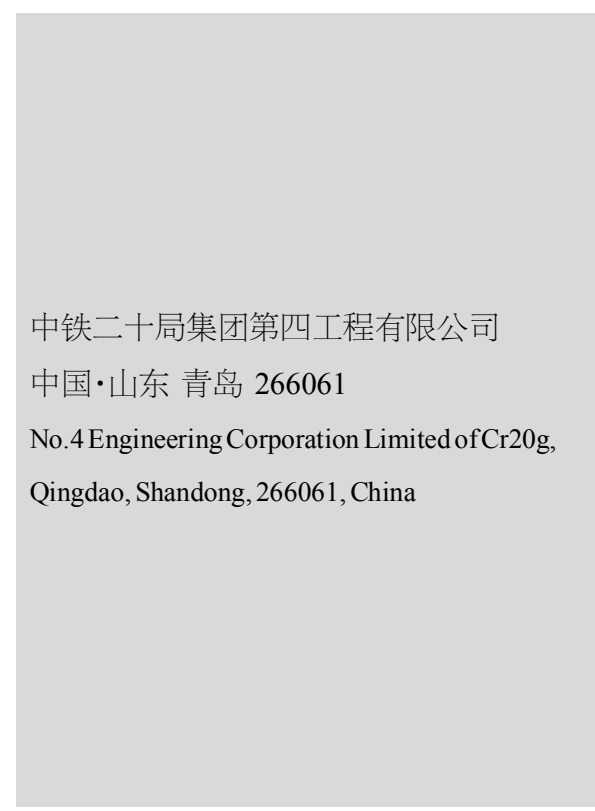

【摘要】随着人们对绿色建筑设计要求的不断提高, 相关专家和研究人士也逐渐开始关 注和重视环境的污染问题和破坏问题。建筑工程在施工的过程中,造成环境污染问题和资 源的破坏问题都是不可避免的。对于这些首要解决的问题,需要对建筑施工进行科学性的 分析, 大力推进绿色建筑的设计方。因此, 论文主要基于 BIM 技术对绿色建筑设计展开了 深入的分析与探究。

【Abstract】With the continuous improvement of people's requirements for green building design, relevant experts and researchers have gradually begun to pay attention to the pollution and destruction of the environment. During the construction process, the problems of environmental pollution and the destruction of resources are inevitable. For these primary problems, it is necessary to analyze the construction scientifically and vigorously promote the designers of green buildings. Therefore, this article mainly based on BIM technology to carry out in-depth analysis and exploration of green building design.

【关键词】BIM 技术; 绿色建筑设计

【Keywords \BIM technology; green building design

[DOI】10.36012/etr.v2i4.1646

\section{1 引言}

目前, 中国资源紧缺和环境污染问题越来越严重,致使绿 色环保和节能减排成为社会发展理念。绿色建筑设计理念的 出现引起了社会和人们的支持和呼吁。绿色建筑设计是在建 筑与环境相协调的基础上开展自然生态系统的良性循环。随 着生活水平的提高, 以及绿色环保意识的增强, 人们对建筑绿 色环保设计的要求也越来越高。因此, 建筑企业一定要将 BIM 技术应用于绿色建筑设计中, 以此来更好地解决建筑设计过 程中所出现的各种问题, 确保建筑工程可以顺利地开展。

\section{BIM 技术的特点}

\section{1 可以有效实现绿色建筑设计方案的可视化}

近些年, 建筑的形状与结构发生了多样化的发展, 导致平 面设计图纸中为建筑设计提供的信息数据具有一定的限制, 虽然可以表明整体建筑的构造情况，但是对图纸进行观察和 制作的过程中仍然存在一定的难度系数。通过 BIM 技术自身 所具有可视化的特点, 应用在绿色建筑设计中可以起到显著 的效果, 传统的二维平面图纸仅是在图纸表面上标注各个构 件的数据信息, 难以将三维效果、安装进度、安装效果直接展
示出来。而 BIM 技术可以让设计者直接按照相关数据信息建 立仿真模型, 在人们的眼前展示出更加直观的设计效果, 而且 构件所处的位置及各个构件之间的关系也可以直观地展现出 来, 可以有效提高工程施工的效率, 同时还可以降低建筑施工 的成本,避免资源浪费。

\section{2 可实现设计、施工及运营各阶段信息共享}

与传统二维平面技术相比, BIM 三维技术不仅可以使绿 色建筑设计的质量得到进一步提高，还可以保障后期施工与 安装工程更加科学、合理。通过 BIM 技术对三维模型进行构 建, 对绿色建筑的设计到安装进行清晰化的了解, 并且也可以 及时了解安装以后的运维维护信息,如施工设备信息、材料信 息等。另外,还可以实时地将绿色建筑设计与施工进度展示出 来。这样一来,在所有阶段中都可以实现资源信息共享, 为整 个绿色建筑工程的开展提供有力的条件。

\section{3 利于提高不同专业设计人员工作的协调性}

对于目前建筑工程的不断发展与扩大，采用传统的绘图 技术已经无法将建筑工程的信息全部表现出来，无法对整个 建筑工程的施工进度和整体的发展情况进行全面的分析和判 断。但是通过利用 BIM 技术可以构建完善的建筑信息模型, 
土木建筑工程 Civil and Architectural Engineering

可以将有关建筑的信息都完全地表达出来。通过采用 BIM 技 术还可以对各个专业进行协调配置, 可以将任意几种专业协 调到统一合作的模型中，这样利于提高不同专业设计人员的 工作效率, 加强对设计信息的交流和传递, 可以有效避免施工 与设计之间出现碰撞, 可以最大限度地提高绿色建筑设计过 程中的质量和效率,进而推动绿色工程顺利的开展。

\section{BIM 技术在绿色建筑设计方面的应用}

\section{1 优化设计方案}

在整个工程建设的过程中，设计阶段是非常关键的一个 环节，通过优化设计方案可以有效地提高整个建筑工程的质 量, 提高工作人员的工作效率。优化设计方案可以从多个方面 加强对建筑基础结构的优化和调整。在项目前期的设计工作 中, 项目投资会给整个工程造成严重的影响。因此, 在对项目 进行优化的过程中需要加强注重对投资成本的优化 ${ }^{[1]}$ 。通过 在设计方案中应用 BIM 技术可以将优化项目的作用充分地 发挥出来, 尤其是在优化设计方案上, 可以在为设计师提供便 利的同时, 还可以有效控制建筑工程的成本, 提高建筑企业的 经济效益。除此之外,利用 BIM 技术也可以对整个工程的实 际情况进行及时的监督和控制, 并对工程施工过程中的资金、 质量等内容进行综合性的控制和管理。

\section{2 节能及能源利用}

目前中国虽然有非常丰富的能源，但是占有量并不是很 高。采用绿色建筑可以有效地提高资源的利用率, 减少对人体 的伤害和对环境的污染, 增强建筑的生态性能, 同时也可以起 到节能减排的作用。通过查阅资料, 开展市场调研发现, 为了 更好地实现建筑节能优化，建筑工程在建设过程中需要采用 节能材料, 具体节能材料及其蓄热系数如表 1 所示。从人力资 源角度分析可知, BIM 技术的参数化后可以根据各种分析结 构做出及时的反应。对于建筑工程的质量、进度及施工成本等 相关信息都可以采用 BIM 技术对相关信息进行实时的获取。 BIM 技术所包含的数据信息都非常的真实和丰富，并且应用 在工程建设软件中可以为其提供有效、可靠的数据, 并且还可 以有效地保障各个数据的真实性和实效性 ${ }^{[2]}$ 。BIM 技术在应用 的过程中可以将各种数据快速直接地计算出来, 降低了人工 计算的工作量, 节约了人力、物力和精力, 提高工作人员的工 作效率。同时,在建设过程中还可以更好地解放人力, 同时还 可以有效地保障信息数据的真实性和可靠性, 可以为绿色建 设设计提供有力的依据。

\section{3 节材及材料利用}

在建筑材料的利用上, 通过采用绿色建筑可以有效地提
表 1 几种节能材料及其蓄热系数 $\left[\mathrm{W} \cdot\left(\mathrm{m}^{2} \cdot \mathrm{K}\right)^{-1}\right]$

\begin{tabular}{c|c|c|c|c|c}
\hline 屋顶 & $\begin{array}{c}\text { 蓄热 } \\
\text { 系数 }\end{array}$ & 外墙 & $\begin{array}{c}\text { 蓄热 } \\
\text { 系数 }\end{array}$ & 门 & $\begin{array}{c}\text { 蓄热 } \\
\text { 系数 }\end{array}$ \\
\hline $\begin{array}{c}\text { 玻璃棉毛 }(90 \mathrm{~mm}) \\
\text { 泡沫玻璃 } \\
(90 \mathrm{~mm})\end{array}$ & 0.340 & 0.704 & $\begin{array}{c}\text { 单层实体 } \\
\text { 木制门 }\end{array}$ & 1.972 \\
\hline $\begin{array}{c}\text { 无机轻集料憎水 } \\
\text { 型膨胀岩保温板 } \\
(90 \mathrm{~mm})\end{array}$ & 3.640 & $\begin{array}{c}\text { A 级防火轻质 } \\
\text { 泡沫混凝土保 } \\
\text { 温板 }(90 \mathrm{~mm})\end{array}$ & 0.440 & $\begin{array}{c}\text { 双层实体 } \\
\text { 木制门 }\end{array}$ & 1.3 \\
\hline $\begin{array}{c}\text { A 级防火轻质泡 } \\
\text { 沫混凝土保温板 } \\
(90 \mathrm{~mm})\end{array}$ & 0.049 & $\begin{array}{c}\text { 无机轻集料憎 } \\
\text { 水型膨胀岩保 } \\
\text { 温板 }(90 \mathrm{~mm})\end{array}$ & 3.640 & - & - \\
$\begin{array}{c}(9) \\
\text { 憎水膨胀珍珠岩 } \\
\text { 制品 }(120 \mathrm{~mm})\end{array}$ & 1.859 & - & - & - & - \\
\hline
\end{tabular}

高材料的利用率, 降低资源的浪费率。对于建筑行业来讲, 耗 费的资源量非常大, 非常容易造成环境污染。从环保角度上分 析, 如果在建筑工程施工的过程中可以对建筑废弃物进行合 理利用, 可以有效解决资源浪费、环境污染的问题。并且, 在绿 色建筑工程设计中, 还可以通过应用一些轻质型的建筑材料, 可以有效地提高建筑材料的环保性, 更好地响应国家绿色环 保的号召。在绿色建筑设计中一定要严格控制施工原材料的 质量, 加强重视施工材料的采购工作, 在挑选材料的时候一定 要选择高环保, 可以循环利用、可再生的建筑材料 ${ }^{[3]}$ 。由于材 料采购的费用占所有费用的 $80 \%$, 故在采购材料之前要根据 图纸的要求制订一个完善的采购计划, 可以利用招标的方式 进行材料的选取。同时, 还可以利用 BIM 技术将其自身强大 的性能充分地展示出来, 工作人员可以采用 BIM 技术软件模 型, 对绿色建筑材料的品种分析和判断, 并对材料的重量和质 量进行篮选, 确保材料的各个性能都可以达到标准。另外, 还 需要对可循环利用的材料进行仔细的检查, 确保材料符合节 能标准, 从而为绿色建筑设计工作的顺利开展奠定基础。

\section{4 结语}

综上所述, 随着中国科学技术的不断创新与发展, BIM 技 术已经被广泛地应用到绿色建筑设计中, 并取得了非常显著 的效果。BIM 技术不仅能够保障绿色建筑工程的施工质量, 还 可以提高整个工程的施工效率, 使信息高效流通。同时, BIM 技术还可以节省建筑工程建设过程中的资源, 从而提高房屋的 环保性能, 为实现精准服务的绿色建筑设计提供有力的依据。

\section{参考文献}

[1]关剑.对基于 BIM 技术的绿色建筑设计应用的几点探讨 [J]. 房 地产导刊,2019(12):212.

[2]孙陈俊妍, 周根, 葛宇佳, 等.BIM 技术在可持续绿色建筑全寿 命周期中的应用研究[J].项目管理技术,2017,15(2):65-69.

[3]刘广周.对基于 BIM 技术的绿色建筑设计应用的几点探讨 $[\mathrm{J}]$. 建筑与装饰, $2017(8): 23+25$. 